\title{
Gene cassettes encoding resistance to quaternary ammonium compounds: a role in the origin of clinical class 1 integrons?
}

\author{
Michael R Gillings ${ }^{1}$, Duan Xuejun ${ }^{1,2}$, Simon A Hardwick ${ }^{1}$, Marita P Holley ${ }^{1}$ \\ and HW Stokes ${ }^{3}$ \\ ${ }^{1}$ Department of Biological Sciences, Macquarie University, Sydney, New South Wales, Australia; ${ }^{2}$ School of \\ Energy and Environmental Engineering, Zhongyuan University of Technology, Zhengzhou, China and \\ ${ }^{3}$ Department of Chemistry and Biomolecular Science, Macquarie University, Sydney, New South Wales, Australia
}

\begin{abstract}
DNA sequencing, phylogenetic and mapping studies suggest that the class 1 integron found in pathogens arose when one member of the diverse family of environmental class 1 integrons became embedded into a Tn402 transposon. However, the timing of this event and the selective forces that first fixed the newly formed element in a bacterial lineage are still unknown. Biocides have a longer use in clinical practice than antibiotics, and a qac (quaternary ammonium compound) resistance gene, or remnant thereof, is a normal feature of class 1 integrons recovered from clinical isolates. Consequently, it is possible that the initial selective advantage was conferred by resistance to biocides, mediated by qac. Here, we show that diverse qac gene cassettes are a dominant feature of cassette arrays from environmental class 1 integrons, and that they occur in the absence of any antibiotic resistance gene cassettes. They are present in arrays that are dynamic, acquiring and rearranging gene cassettes within the arrays. The abundance of qac gene cassettes makes them a likely candidate for participation in the original insertion into $\mathrm{Tn} 402$, and as a source of a readily selectable phenotype. More broadly, the increasing use of qac and other biocides at the present time seems likely to promote the fixation of further novel genetic elements, with unpredictable and potentially adverse consequences for human health and agriculture.
\end{abstract}

The ISME Journal (2009) 3, 209-215; doi:10.1038/ismej.2008.98; published online 16 October 2008

Subject Category: evolutionary genetics

Keywords: antibiotic resistance; biocide resistance; evolution; integron; lateral gene transfer; qac

\section{Introduction}

The spread of antimicrobial resistance genes throughout human-dominated ecosystems has generated a world-wide crisis in the management of infectious disease (Davies, 2007). Plasmid, transposon and integron-borne resistance to antibiotics now occurs in the majority of Gram-negative pathogens isolated from hospitals and animal production facilities (Goldstein et al., 2001; Ebner et al., 2004; Essen-Zandbergen et al., 2007). Understanding the origin of resistance determinants and the mobile elements that disseminate them is critical in attempting to control future recruitment and spread of resistance genes.

The class 1 integron is an important platform for recruitment of resistance genes. Class 1 integrons

Correspondence: MR Gillings, Department of Biological Science, Macquarie University, Sydney, New South Wales 2109 Australia. E-mail: michael.gillings@mq.edu.au

Received 10 June 2008; revised 3 September 2008; accepted 9 September 2008; published online 16 October 2008 encode an integrase, IntI1, which inserts circular gene cassettes at an integron-associated recombination site, attI1, and then drives their expression with an adjacent promoter, $\mathrm{P}_{\mathrm{c}}$ (Stokes and Hall, 1989; Hall and Collis, 1995; Hall et al., 1999). The diversity of gene cassettes present in natural environments is enormous, amounting to many thousands of different cassettes even in small areas $\left(<50 \mathrm{~m}^{2}\right)$, each encoding novel functions (Stokes et al., 2001; Michael et al., 2004; Boucher et al., 2007). These cassettes can be sampled by class 1 integrons (RoweMagnus et al., 2002), whereby selection retains any that confer an advantageous phenotype, thus accounting for the accumulation of resistance cassettes by the class 1 integrons found in pathogens. The location of class 1 integrons on various mobile elements facilitates their rapid spread into new species (Liebert et al., 1999; Partridge et al., 2001). The ability of class 1 integrons to access diverse genes and their inherent mobility has led to their preeminent role in the spread of resistance determinants through human ecosystems. The typical class 1 integron recovered from clinical contexts is 
embedded in a plasmid or transposon, and generally carries from 1 to 6 gene cassettes, primarily encoding antibiotic resistance (Recchia and Hall, 1995; Partridge et al., 2001). We will refer to these as 'clinical' class 1 integrons.

Recently, class 1 integrons have been recovered from diverse environmental samples. These integrons differ from those in clinical contexts, in that they are chromosomal, and do not carry antibiotic resistance cassettes. There is good evidence to suggest that the clinical class 1 integron originated when one of these chromosomal class 1 integrons mobilized into a Tn402-like transposon (Stokes et al., 2006; Gillings et al., 2008a). Extant clinical class 1 integrons have a series of conserved features and sequences that strongly suggest such a single common origin. For instance, the integron-integrase gene (intI1) is essentially identical in all clinical class 1 integrons, and the left hand end of all clinical class 1 integrons contains a conserved noncoding sequence that terminates in a 25 -bp sequence (IRi), which is an inverted repeat of another sequence (IRt) located at the right hand end of most extant clinical class 1 integrons (Brown et al., 1986, 1996, Figure 1a). These inverted repeats define the structural boundary of the clinical class 1 integron, as they are the recognition sites required for Tn402mediated transposition (Kholodii et al., 1995).

The immediate common ancestor of all clinical class 1 integrons was probably similar to a Tn402like transposon, consisting of (in order) IRi, intI1, attI1, gene cassette(s) and their associated recombination sites (59-base elements, or attC), a qacE (quaternary ammonium compound E) cassette (which encodes resistance to biocides), a complete tni transposition module and IRt (Stokes et al., 2006 and references therein, Figure 1a). The majority of extant class 1 integrons from clinical contexts carry various modifications to the right hand end of this ancestral integron, including deletions in qacE, incorporation of a sulfonamide resistance gene (sull) and partial deletions of the tni module (Liebert et al., 1999; Partridge et al., 2001; Stokes et al., 2006).

Insertion of a chromosomal class 1 integron into a Tn402-like transposon followed by lateral transfer into a human commensal or pathogen provided a route into human ecosystems, but how was this rare event preserved by selection? Because qacE is an apparently ancestral feature of clinical class 1 integrons, we have suggested that it was the possession of a qacE gene cassette that provided the selective advantage for the newly generated Tn402-integron in the human environment, and that it did so by conferring resistance to biocides (Gillings et al., 2008a). If this were the case, then class 1 integrons containing qac gene cassettes should be recoverable from environmental samples. Further, these integrons should not necessarily carry antibiotic resistance cassettes. Here, we show that diverse $q a c$-encoding gene cassettes can be readily recovered using metagenomic DNA extracted from biofilms and sediments, and that qac cassettes are a common feature of environmental class 1 integrons.

\section{Materials and methods}

Extraction of DNA from environmental samples Sediment and biofilm samples $(n=51)$ were all obtained from a range of sites in the metropolitan area of Sydney, NSW, Australia. Samples included stream sediments from Cowan Creek, North Turramurra (RA: Hardwick et al., 2008), biofilms from Mars Creek, North Ryde (BF: Gillings et al., 2008b), contaminated soil from Balmain (BAL; Yeates and Gillings, 1998), biofilms from a groundwater treatment plant in Botany (OR: Gillings et al., 2006) and a

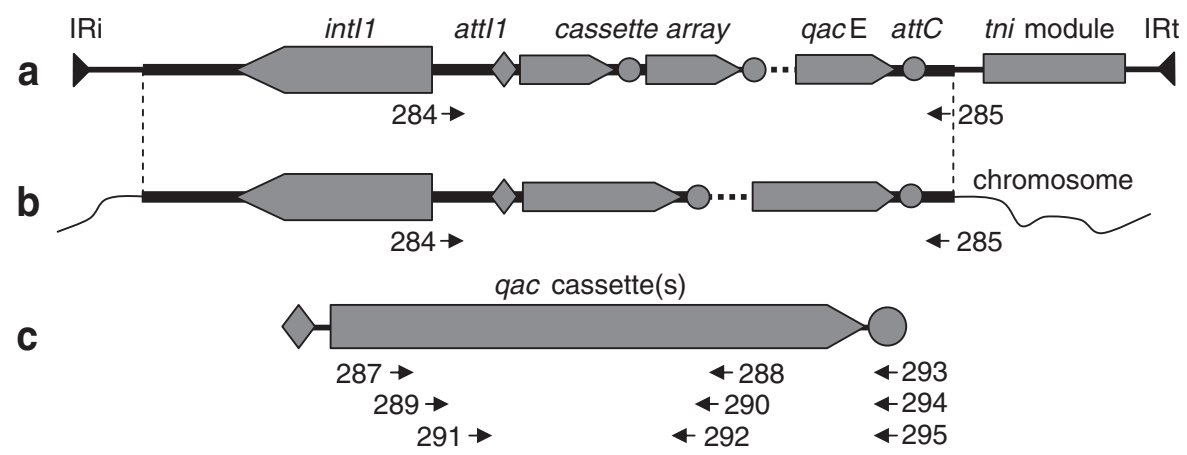

Figure 1 Schematic representation of class 1 integrons and their gene cassettes, showing the locations of primers used to amplify integron constituents. (a) Transposon Tn402 that includes a class 1 integron. This element carries the terminal inverted repeats (IRi and IRt), a class 1 integron integrase gene (intI1), a primary recombination site (attI; small diamond), a cassette array (usually encoding antibiotic resistance) with each cassette bounded by secondary recombination sites (attC; circles), a cassette for quaternary ammonium compound resistance ( $q a c E$ ) and a transposition module (tni). (b) An environmental class 1 integron embedded into a bacterial chromosome carries the integrase gene, flanking regions and a cassette array that does not usually encode antibiotic resistance. The primer combination MRG284/285 amplifies complete arrays from both these kinds of class 1 integrons. Dotted vertical lines denote the extent of sequence homology between (a) and (b), and define the boundaries of the class1 integron (Gillings et al., 2008a, b). (c) A representation of a qac gene cassette. Primers in the 287-295 series amplify coding regions of different qac groups, and qac genes associated with gene cassettes. Differences in the product sizes allow identification of each group (see Table 1, Figure 3). 
biofilm from a suburban pond in Seven Hills. None of the sampling sites was downstream from hospitals, sewage treatment works or animal production facilities. Samples were stored at $4{ }^{\circ} \mathrm{C}$ before extraction of DNA, normally within $24 \mathrm{~h}$. DNA was extracted from $400 \mathrm{mg}$ subsamples using bead beating in a FastPrep machine (Bio 101, Morgan Irvine, CA, USA) (Yeates and Gillings 1998). DNA yield was assessed using agarose electrophoresis.

\section{PCR amplifications from environmental DNA and pure cultures}

An internal fragment of the class 1 integronintegrase gene, intI1, was amplified using primers HS463a and HS464 as described (Table 1, Stokes et al., 2006). Cassette arrays associated with class 1 integrons were amplified using primers MRG284 and MRG285 (Figure 1 and Table 1). PCRs were performed in $50 \mu \mathrm{l}$ reactions with GoTaq DNA polymerase (Promega, Madison, WI, USA) in the buffer supplied with the enzyme, $25 \mathrm{pmol}$ of each primer and a final $\mathrm{Mg}^{2+}$ concentration of $1.5 \mathrm{mM}$. PCRs were carried out using an Omn-E thermocycler (Hybaid, Middlesex, UK) with the following cycling programs: $94{ }^{\circ} \mathrm{C}$ for $3 \mathrm{~min}$ (one cycle); $94{ }^{\circ} \mathrm{C}$ for $30 \mathrm{~s}$, 60 or $65{ }^{\circ} \mathrm{C}$ for $30 \mathrm{~s}, 72^{\circ} \mathrm{C}$ for $90 \mathrm{~s}$ (35 cycles); $72{ }^{\circ} \mathrm{C}$ for $5 \mathrm{~min}$ (one cycle). Positive controls were used in every amplification. These included a typical clinical class 1 cassette array located on plasmid R388 (accession no. BR000038) and a representative environmental cassette array from the chromosomal class 1 integron in Thauera sp B4 (accession no. EU327991) (Gillings et al., 2008a).

Genes in the qac family were amplified using primers MRG287/MRG288 (qacG/E2 homologs, positive control BF12_C8, accession no. FJ172380) or MRG289/MRG290 (qacH homologs, positive control BF12_C5, accession no. FJ172378) or MRG291/292
(qacE1 homologs, positive control BF13_C3, accession no. FJ172384; see Table 1). PCR conditions were as above except that all six qac primers were used in a multiplex reaction containing $10 \mathrm{pmol}$ of each primer and an annealing temperature of $55^{\circ} \mathrm{C}$ was used. To confirm that the targeted qac genes resided in gene cassettes, the forward primers used above were paired with attC-specific reverse primers, using the same conditions and positive controls (Table 1). Small subunit ribosomal RNA genes (16S rDNA) were amplified using primers $\mathrm{f} 27$ and r1492 (Lane, 1991).The efficiency of PCR was assessed by electrophoresis on 2 or $2.5 \%$ agarose gels.

\section{Real-time quantitative PCR}

Real-time quantitative PCR assays were targeted at 16S rDNA, intI1 and qac gene cassettes from biofilm samples, following the methods given in Hardwick et al. (2008). Pseudomonas stutzeri Q (Holmes et al., 2003) was used as a positive control for 16S rDNA, plasmid R388 was used as a positive control for intI1 and plasmid BF12-5 was used as a positive control for qac gene cassettes. Standards of known copy number were prepared and run in triplicate to generate standard curves. Assays were performed in an ABI7700 Prism sequence detector (Applied Biosystems, Foster City, CA, USA). Each environmental DNA sample was tested in triplicate using $25 \mathrm{ng}$ of DNA template. Programming, detection and analysis were performed as described previously (Hardwick et al., 2008).

Cloning of PCR products

PCR products were purified using the Wizard PCR Preps DNA Purification System (Promega) and ligated into T-tailed TOPO plasmid vectors (Invitrogen, Carlsbad, CA, USA). Transformation into

Table 1 Details of PCR primer combinations

\begin{tabular}{|c|c|c|c|}
\hline Primers & Sequence $\left(5^{\prime}-3^{\prime}\right)$ & Target & Size $(b p)$ \\
\hline HS463a & F CTGGATTTCGATCACGGCACG & intI1 & 473 \\
\hline HS464 & R ACATGCGTGTAAATCATCGTCG & & \\
\hline MRG284 & F GTTACGCCGTGGGTCGATG & Class 1 integron & Variable $^{\mathrm{a}}$ \\
\hline MRG285 & R CCAGAGCAGCCGTAGAGC & Cassette array & \\
\hline MRG284 & F GTTACGCCGTGGGTCGATG & Class 1 integron & Variable \\
\hline MRG286 & R CCACTGCCGCCATTGGGC & cassette array & \\
\hline MRG287 & F TTGGTTATTTCTGGCTACG & qacG family & 281 \\
\hline MRG288 & R CGCTGATAATGAAGCCGAC & & \\
\hline MRG289 & F AAGTCCAGCCATGGATTC & $q a c \mathrm{H}$ family & 235 \\
\hline MRG290 & R GGCGACGCCACTGACG & & \\
\hline MRG291 & F AGCTTGCCCCTTCCGC & gac E family & 193 \\
\hline MRG292 & R AGCCCCATACCTACAAAG & & \\
\hline MRG287 & F TTGGTTATTTCTGGCTACG & qacG cassettes & 354 \\
\hline MRG293 & R TCGACGGAATGGTTAGATG & & \\
\hline MRG289 & F AAGTCCAGCCATGGATTC & qacH cassettes & 301 \\
\hline MRG294 & R GCTTGAATGAATTGTTAGATG & & \\
\hline MRG291 & F AGCTTGCCCCTTCCGC & qacE cassettes & 277 \\
\hline MRG295 & R TGCAACGACTTGTTAGATG & & \\
\hline
\end{tabular}

aProduct sizes vary according to number and type of gene cassettes in the amplified array. 
chemically competent Escherichia coli OneShot TOP10 cells (Invitrogen) was carried out in accordance with the manufacturer's instructions. Twenty-four random clones were chosen from each environment and screened for the presence of inserts using PCR with the original primers on boiled colony lysates. These were prepared by suspending one colony in $200 \mathrm{ul}$ of sterile water, incubation at $99^{\circ} \mathrm{C}$ for $10 \mathrm{~min}$, snap chilling on ice and centrifugation for $5 \mathrm{~min}$ at $12000 \mathrm{~g}$. A 1-ul aliquot of the supernatant was used as a template for PCR.

\section{DNA sequencing of selected clones}

Plasmids were purified from $5 \mathrm{ml}$ overnight liquid cultures using the Wizard Plus Minipreps DNA Purification System (Promega) as per the manufacturer's instructions. Sequencing reactions contained $\sim 200 \mathrm{ng}$ of plasmid DNA and 4 pmol of pcrfnew forward or pcrR30 reverse vector primers (Gillings et al., 2005). Sequencing was performed on an ABI 3130xl Genetic Analyzer using BigDye v 3.1 chemistry (Applied Biosystems). Sequencing reactions, unincorporated dye-terminator removal and capillary electrophoresis were carried out at the Macquarie University DNA Analysis Facility. Sequences were interrogated by searching against nonredundant nucleotide or protein databases using blastn and blastx algorithms (http://www.ncbi.nlm.nih. gov/BLAST/). Phylogenetic and molecular evolutionary analyses were conducted using the bioinformatics tools available through Biomanager, on the ANGIS web site (http://www.angis.org.au/).

\section{Data deposition}

DNA sequences reported in this study were deposited with GenBank as accession numbers FJ172371FJ172422.

\section{Results}

Primers were designed to specifically amplify class 1 integron gene cassette arrays. Primer sequences were based on an alignment of the termini of known chromosomal class 1 cassette arrays and arrays carried on plasmids or transposons from clinical contexts (Gillings et al., 2008a). These primers amplify arrays from chromosomal integrons and Tn402-like elements, by targeting a region in attI1 proximal to the array (MRG284), and a region beyond the final attC site in the array (MRG285) (Figure 1). PCR assays using these primers produced complex amplification patterns from metagenomic DNA, generating multiple bands between 400 and $2000 \mathrm{bp}$ from individual samples, suggesting that multiple class 1 gene cassette arrays were present. All 51 sediment and biofilm samples tested generated products in the assay (data not shown). PCR products from selected amplifications were cloned and DNA sequenced to determine the identity of cassettes within the arrays.
Fifty-two cassette array clones from a total of 18 independent environmental samples were sequenced. The arrays contained 1-3 cassettes bounded by characteristic attC sites, but in other respects were not typical of class 1 cassette arrays from clinical isolates. Two of the 70 cassettes detected encoded potential antibiotic resistance determinants. Both of these were aadA10 cassettes with greater than $99.9 \%$ nucleotide identity to resistance cassettes commonly found in pathogens, suggesting they originated from a human or animal source. Two further cassettes, each from independent samples, encoded putative cupin barrel proteins (Dunwell et al., 2001). Sequence homology placed them in COG1917, Cupin 2 barrel proteins, a family that has never previously been associated with integrons. The function of this family is currently unknown. Thirty-nine of the cassettes encoded conserved hypothetical proteins, or contained open reading frames for which no homology could be found in sequence databases. Such cassettes are typical of chromosomal integrons from environmental samples. The final 27 cassettes all encoded efflux pumps of the qac family, such that in total, about $50 \%$ of the class 1 arrays from environmental samples carried a qac cassette (Supplementary Table 1).

Comparison of the cassette arrays showed that the same cassette ( $>99 \%$ nucleotide sequence homology) could be found in a number of different contexts and located adjacent to a number of different cassettes. The same qacG/E2 cassette could be found as the sole cassette within an array, or adjacent to one of the three different cassettes (Figure 2). Similarly, cassettes encoding qacH, cupin barrel proteins and hypothetical proteins could also be found in a number of different contexts (Figure 2). Further examples of this phenomenon (Supplementary Table 1) suggested that there is a dynamic process of cassette acquisition and rearrangement in these arrays.

Phylogenetic analysis of the qac cassettes revealed three clades, corresponding to groups commonly annotated as qacE1, qacG/E2 and qacH, respectively. Alignments between these cassettes were used to design primers to specifically amplify the coding regions of each group, and to distinguish between the various groups based on amplicon size (Table 1 and Figure 1). To assess the distribution and abundance of each qac group in the natural environment, these primers were used in a multiplex PCR on all biofilm and stream sediment DNA samples $(n=48)$. Bands of the expected size(s) amplified from the majority of samples, although the strength of amplification varied. In addition, the relative proportions of the three different qac groups were different in different samples. For instance, some samples generated three bands of equal strength, suggesting similar concentrations of the three qac groups, whereas other samples were dominated by one of the qac groups (Figure 3). 

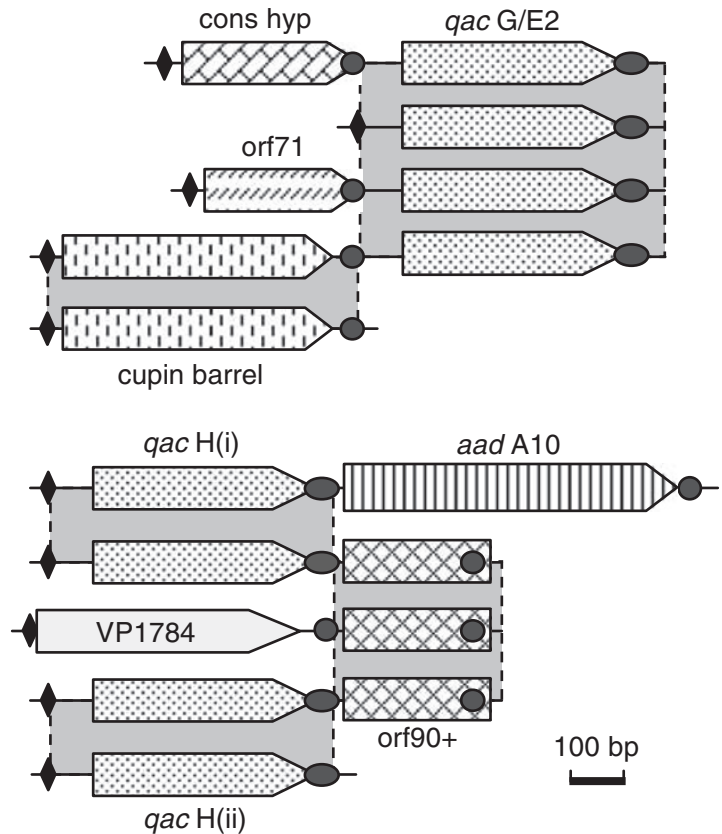

Figure 2 Schematic maps of class 1 integron cassette arrays from environmental samples. Maps were constructed on the basis of sequence data for cassette arrays amplified from environmental samples using primers MRG284/285 (Table 1 and Supplementary Table 1). Symbols are as follows: black diamonds, attI1; gray circles, attC (59-be); block arrows are open reading frames within cassettes, labeled with gene names. All qac genes are stippled; gray shading between cassettes denotes greater than $99 \%$ nucleotide sequence homology. The two different sets of $q a c \mathrm{H}$ cassettes (i and ii) in the bottom part of the figure exhibit $96 \%$ nucleotide sequence homology. Individual qac gene cassettes can be found in a variety of different contexts, suggesting a dynamic process of cassette acquisition and rearrangement in these arrays.

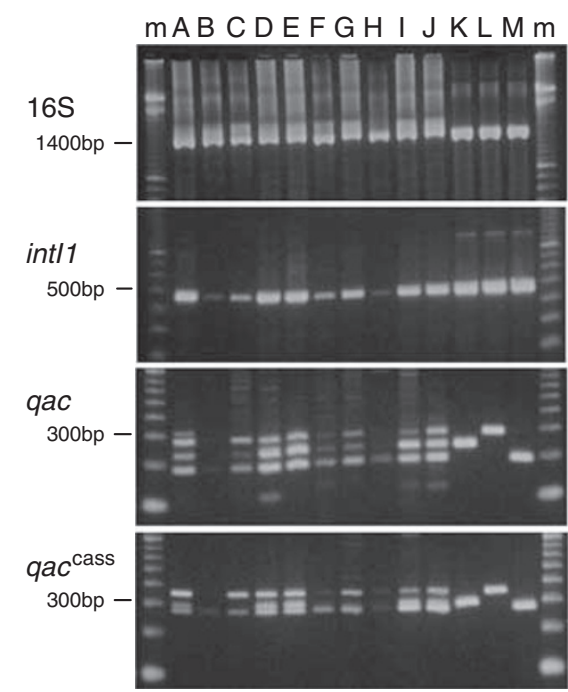

Figure 3 Representative PCR amplifications from metagenomic DNA samples. A series of environmental DNA samples were used as a template for amplification of $16 \mathrm{~S} \mathrm{rDNA}$ and the integron-integrase gene, intI1. Multiplex PCR was used to amplify coding regions of the qac E1, G/E2 and $\mathrm{H}$ families, and to amplify these same regions from within gene cassettes ( $q^{\text {cass }}{ }^{\text {cas }}$. Tracks are from the following environmental samples: (A) BF7, (B) BF2, (C) BF4, (D) BF11, (E) BF12, (F) BF16, (G) BF13, (H) BF17, (I) BF15 and (J) BF24. Tracks (K-M) are positive controls as detailed in Materials and methods section, $(\mathrm{m})=100 \mathrm{bp}$ molecular weight ladder. Sizes are given at the left hand side.
The overall intensity of response generated in qac PCR correlated extremely well with the response generated by amplification of the same samples with intI1 primers (Figure 3). However, this variation was not correlated with the intensity of $16 \mathrm{~S}$ rDNA amplifications (for example, tracks $\mathrm{B}$ vs $\mathrm{C}$ in Figure 3). This suggested that there was variation in the abundance of class 1 integrons between samples, but that the proportion of class 1 integrons that contained qac cassettes did not change. To confirm that the qac PCR products did represent qac coding regions embedded within gene cassettes, a second multiplex PCR was performed using the forward qac primers and a set of three reverse primers anchored in the attC sites specifically associated with each qac group (Figure 1). The intensities of bands generated in this assay matched those of the intI1 and qac assays (Figure 3). The best explanation for the PCR results, in conjunction with the sequencing results, is that a large and near constant proportion of class 1 integrons from environmental samples carried qac gene cassettes.

To further investigate the abundance and diversity of class 1 cassette arrays and their associated qac gene cassettes, we performed real-time quantitative PCR directed at intI1 and qac gene cassettes in all the biofilm samples $(n=24)$. Numbers of cells containing intI1 were normalized with respect to 16S rDNA quantitative PCR performed on the same samples (Hardwick et al., 2008). On the basis of this analysis, between 1 and $9 \%$ of cells in the biofilms were intI1 positive (mean \pm s.e. $=4.5 \pm 0.55 \%$ ). The proportion of cells in the various biofilms that carried qac gene cassettes was approximately half of this value (mean \pm s.e. $=2.2 \pm 0.59 \%$ ). These data agree with those from the sequencing analysis, suggesting that about $50 \%$ of intI1-positive cells from environmental samples carry a qac gene cassette.

\section{Discussion}

It is broadly accepted that resistance to antimicrobial agents is one of the most important medical issues facing humankind (Davies, 2007). Lateral gene transfer has played a critical role in the dissemination of these resistance determinants, but we still have little information about the sources of resistance genes and their mobile vectors. Examination of enterobacterial isolates collected during the 1920s found no antibiotic resistance genes (Datta and Hughes, 1983; Hughes and Datta, 1983); so clearly, over the last 90 years such genes have been repeatedly sampled from the vast reservoir of microbial diversity that resides in natural environments. Studying the pool of potential resistance genes and mobile vectors could help predict future threats posed by lateral gene transfer events (D'Costa et al., 2006). 
Previous examination of the diversity of intI1 in environmental samples showed that the ancestor of the class 1 integrons now circulating in human commensals and pathogens was probably an integron residing on the chromosome of a $\beta$-proteobacterial species. Under this model, the chromosomal class 1 integron was inserted into a Tn402 transposon, which then found its way into a human commensal or pathogen (Stokes et al., 2006; Gillings et al., 2008a). It was suggested that the possession of a qac gene cassette provided a selective advantage for this novel DNA element (Gillings et al., 2008a) by conferring resistance to the qac used as disinfectants in hospitals from the 1930s onwards (Russell, 2002). We now have strong supporting evidence for this hypothesis.

Diverse qac gene cassettes that are resident in class 1 integron cassette arrays can be readily recovered from environmental samples. These qac cassettes were generally not associated with any known antibiotic resistance cassettes, as might be expected if the arrays we detected were being shed from hospitals, animal production facilities or sewage systems. About half of all class 1 integron arrays from environmental samples carry qac cassettes, such that they are the most common cassette type recovered. Further, individual qac cassettes occur in many different contexts, suggesting a dynamic process of cassette acquisition and rearrangement in these arrays. Consequently, insertion of any individual environmental class 1 integron into Tn 402 was more likely than not to carry a qac gene cassette in its array. The widespread distribution of diverse qac cassettes hints at a general role for these efflux pumps in environmental bacteria. It is thought that efflux pumps defend against toxins in natural ecosystems (Gilbert and McBain, 2003), but the natural targets of qac efflux systems are currently unknown.

The rise in resistance to antibiotics is paralleled by a similar trend in resistance to biocides (Higgins et al., 2001; Bjorland et al., 2005), prompting concerns that the increasingly widespread use of biocides enhances the selection and dissemination of antibiotic resistant bacteria (Russell, 2002; Gilbert and McBain, 2003). There are two widely discussed mechanisms by which this coselection might occur. The first of these is the acquisition of generic resistance mechanisms that function to exclude or remove both antibiotics and biocides, such as changes to membrane properties or the acquisition of efflux pumps (Russell et al., 1998; Langsrud et al., 2004; Poole, 2005). The second mechanism involves coselection of antibiotic resistance genes through their linkage with biocide resistance determinants on the same plasmid or transposon. The best examples of such a case are class 1 integrons from clinical contexts, where genes for resistance to qac, or fragments thereof, are often closely linked to gene cassettes encoding antibiotic resistance (White and McDermott, 2001; Gaze et al., 2005).
There is, however, a third mechanism whereby the human use of biocides may promote dissemination of mobile genetic elements and hence resistance genes, and this is by promoting the selection of novel lateral gene transfer events from environmental bacteria. All newly acquired mobile elements and resistance genes ultimately originate from natural environments. Their passage from natural environment either directly into a pathogen or through a human commensal is reliant on (i) an initial, low frequency, transfer event and (ii) selection for any newly acquired phenotype. Consequently, the more selection we apply, the more diverse lateral transfer events become fixed in human ecosystems. These transferred elements can then participate in the rearrangements and further exchanges that typify the mosaic structure of mobile DNAs (Toussaint and Merlin, 2002), multiplying the evolutionary opportunities for interactions between mobile elements, transposases, site-specific recombinases and any associated genes that confer novel phenotypes (Frost et al., 2005; Tetu and Holmes, 2008). Use of biocides may have driven the fixation and spread of the class 1 integron, now responsible for a major part of antibiotic resistance. Current trends for broader and more indiscriminate use of biocides in homes, businesses and hospitals may drive the emergence of new genetic elements, with unpredictable consequences for human welfare.

\section{Acknowledgements}

This study was supported by grants from the Australian Research Council and the National Health and Medical Research Council.

\section{References}

Bjorland J, Steinum T, Kvitle B, Waage S, Sunde M, Heir E. (2005). Widespread distribution of disinfectant resistance genes among staphylococci of bovine and caprine origin in Norway. J Clin Microbiol 43: 4363-4368.

Boucher Y, Labbate M, Koenig J, Stokes H. (2007). Integrons: mobilizable platforms that promote genetic diversity in bacteria. Trends Microbiol 15: 301-309.

Brown NL, Misra TK, Winnie JN, Schmidt A, Seiff M, Silver S. (1986). The nucleotide sequence of the mercuric resistance operons of plasmid R100 and transposon Tn501: further evidence for mer genes which enhance the activity of the mercuric ion detoxification system. Mol Gen Genet 202: 143-151.

Brown HJ, Stokes HW, Hall RM. (1996). The integrons In0, In2 and In5 are defective transposon derivatives. J Bacteriol 178: 4429-4437.

Datta N, Hughes VM. (1983). Plasmids of the same Inc groups in Enterobacteria before and after the medical use of antibiotics. Nature 306: 616-617.

Davies J. (2007). Microbes have the last word. EMBO Rep 8: $616-621$ 
D’Costa VM, McGrann KM, Hughes DW, Wright GD. (2006). Sampling the antibiotic resistome. Science 311: 374-377.

Dunwell J, Culham A, Carter C, Sosa-Aguirre C, Goodenough P. (2001). Evolution of functional diversity in the cupin superfamily. Trend Biochem Sci 26: 740-746.

Ebner P, Garner K, Mathew K. (2004). Class1 integrons in various Salmonella enterica serovars isolated from animals and identification of genomic island SGI1 in Salmonella enterica var. Melagridis. J Antimicrob Chemother 53: 1004-1009.

Essen-Zandbergen A, Smith H, Veldman K, Mevius D. (2007). Occurrence and characteristics of class 1, 2 and 3 integrons in Escherichia coli, Salmonella and Campylobacter spp. in the Netherlands. J Antimicrob Chemother 59: 746-750.

Frost L, Leplae R, Summers AO, Toussaint A. (2005). Mobile genetic elements: the agents of open source evolution. Nat Rev Microbiol 3: 722-732.

Gaze WH, Abdouslam N, Hawkey PM, Wellington EMH. (2005). Incidence of class 1 integrons in a quaternary ammonium compound-polluted environment. Antimicrobial Agents Chemother 49: 1802-1807.

Gilbert P, McBain A. (2003). Potential impact of increased use of biocides in consumer products on prevalence of antibiotic resistance. Clin Microbiol Rev 16: 189-208.

Gillings M, Holley M, Selleck M. (2006). Molecular identification of species comprising an unusual biofilm from a groundwater treatment plant. Biofilms 3: 19-24.

Gillings M, Boucher Y, Labbate M, Holmes A, Krishnan S, Holley $\mathrm{M}$ et al. (2008a). The evolution of class 1 integrons and the rise of antibiotic resistance. J Bacteriol 190: 5095-5100.

Gillings M, Krishnan K, Worden P, Hardwick S. (2008b). Recovery of diverse genes for class 1 integronintegrases from environmental DNA samples. FEMS Microbiol Lett 287: 56-62.

Gillings M, Holley M, Stokes H, Holmes A. (2005). Integrons in Xanthomonas: A source of species genome diversity. Proc Natl Acad Sci USA 102: 4419-4424.

Goldstein C, Lee M, Sanchez S, Hudson C, Phillips B, Register B et al. (2001). Incidence of class 1 and 2 integrases in clinical and commensal bacteria from livestock, companion animals, and exotics. Antimicrob Agents Chemother 45: 723-726.

Hall R, Collis C. (1995). Mobile gene cassettes and integrons: Capture and spread of genes by site-specific recombination. Mol Microbiol 15: 593-600.

Hall RM, Collis CM, Kim M-J, Partridge SR, Recchia GD, Stokes HW. (1999). Mobile gene cassettes in evolution. Ann NY Acad Sci 87: 68-80.

Hardwick S, Stokes HW, Findlay S, Taylor M, Gillings M. (2008). Quantification of class 1 integron abundance in natural environments using real-time quantitative PCR. FEMS Microbiol Lett 278: 207-212.

Higgins CS, Murtough SM, Williamson E, Hiom SJ, Payne DJ, Russel AD et al. (2001). Resistance to antibiotics and biocides among non-fermenting Gramnegative bacteria. Clin Microbiol Infect 7: 308-315.

Holmes A, Holley M, Mahon A, Nield B, Gillings M, Stokes H. (2003). Recombination activity of a distinctive integron-gene cassette system associated with Pseudomonas stutzeri populations in soil. J Bacteriol 185: 918-928.
Hughes VM, Datta N. (1983). Conjugative plasmids in bacteria in the 'pre-antibiotic' era. Nature 302: 725-726.

Kholodii GY, Mindlin SZ, mBass IA, Yurieva OV, Minakhina SV, Nikiforov VG. (1995). Four genes, two ends and a res region are involved in transposition of Tn5053: a paradigm for a novel family of transposons carrying either a mer operon or an integron. Mol Microbiol 17: 1189-1200.

Lane D. (1991). 16S/23S rRNA sequencing. In: Stackebrandt E, Goodfellow M (eds). Nucleic Acid Techniques in Bacterial Systematics. John Wiley and Sons: London, pp 115-175.

Langsrud S, Sundheim G, Holck AL. (2004). Cross resistance to antibiotics of Escherichia coli adapted to benzalkonium chloride or exposed to stress inducers. J Appl Microbiol 96: 201-208.

Liebert C, Hall R, Summers A. (1999). Transposon Tn21, flagship of the floating genome. Microbiol Mol Biol Rev 63: 507-522.

Michael C, Gillings M, Holmes A, Hughes L, Andrew N, Holley $\mathrm{M}$ et al. (2004). Mobile gene cassettes: A fundamental resource for bacterial evolution. Am Nat 164: 1-12.

Partridge S, Recchia G, Stokes H, Hall R. (2001). Family of class 1 integrons related to In4 from Tn1696. Antimicrob Agents Chemother 45: 3014-3020.

Poole K. (2005). Efflux-mediated antimicrobial resistance. J Antimicrobial Chemother 56: 20-51.

Recchia G, Hall R. (1995). Gene cassettes, a new mobile element. Microbiol 141: 3015-3027.

Rowe-Magnus D, Guerot A-M, Mazel D. (2002). Bacterial resistance evolution by recruitment of super-integron gene cassettes. Mol Microbiol 43: 1657-1669.

Russell AD. (2002). Introduction of biocides into clinical practice and the impact on antibiotic resistant bacteria. J Appl Microbiol Symp Suppl 92: 121S-135S.

Russell AD, Tattawasart U, Maillard J-Y, Furr JR. (1998). Possible link between bacterial resistance and use of antibiotics and biocides. Antimicrob Agents Chemother 42: 2151.

Stokes H, Holmes A, Nield B, Holley M, Nevalainen K, Mabbutt B et al. (2001). Gene cassette PCR: Sequence independent recovery of entire genes from environmental DNA. Appl Env Microbiol 67: 5240-5246.

Stokes H, Hall R. (1989). A novel family of potentially mobile elements encoding site-specific gene-integration functions: integrons. Mol Microbiol 3: 1669-1683.

Stokes H, Nesbø C, Holley M, Bahl M, Gillings M, Boucher Y. (2006). Class 1 integrons predating the association with Tn402-like transposition genes are present in a sediment microbial community. $J$ Bacteriol 188: $5722-5730$

Tetu S, Holmes A. (2008). A family of insertion sequences that impacts integrons by specific targeting of gene cassette recombination sites, the IS1111-attc group. J Bacteriol 190: 4959-4970.

Toussaint A, Merlin C. (2002). Mobile elements as a combination of functional modules. Plasmid 47: 26-35.

White DG, McDermott PF. (2001). Biocides, drug resistance and microbial evolution. Curr Opinion Microbiol 4: 313-317

Yeates C, Gillings M. (1998). Rapid purification of microbial DNA from soil for molecular biodiversity analysis. Lett Appl Microbiol 27: 49-53. 